\title{
Aortic aneurysm replacement with Dacron graft in a 34 year old man. How long will the graft last?
}

\author{
B F Khan, S Renton, S T Hussain
}

Postgrad Med J 2003;79:348-349

A unique case of an abdominal aortic aneurysm replacement in a young man of Caribbean descent is presented. The initial working diagnosis was of a mycotic aneurysm, which has recently shown resurgence with intravenous drug abuse. Blood tests and subsequent biopsy ruled that out. There was also a clinical suspicion of a connective tissue abnormality. Histological examination of the aneurysm, skin biopsy, and blood tests also ruled out this possibility. The graft used in this patient was from a new generation of grafts. In the absence of any studies on such grafts, there is the question of long term durability of the graft.

A 34 year old man of Caribbean descent presented to the accident and emergency department with a two week history of upper abdominal pain. The pain was described as a dull ache with no exacerbating or relieving factors. There were no other symptoms and no recent travel abroad. His past medical history included a left inguinal hernia repair and ruptured left Achilles tendon repair. He was a non-smoker with no cardiovascular risk factors. On examination he was a muscular and fit young man. His blood pressure was 160/100 mm Hg and he was apyrexial. Abdominal examination revealed tenderness in the epigastrium. No expansile mass was noted. The rest of the examination was normal.

An urgent abdominal ultrasound scan was performed, which revealed an infrarenal abdominal aortic aneurysm measuring $5.5 \mathrm{~cm}$ in the anteroposterior diameter. The rest of the ultrasound examination was normal. Computed tomography of the thoracic cavity revealed no abnormality of the thoracic aorta. Duplex ultrasound of the lower limb vessels showed no evidence of iliac, femoral, or popliteal aneurysms. His haemoglobin concentration and white cell count, lipid profile, erythrocyte sedimentation rate, C-reactive protein, liver enzymes, plasma proteins, rheumatoid antibodies, antinuclear antibodies, antineutrophil cytoplasmic antibodies, sickle screen test, and serum protein electrophoresis were all normal.

A working diagnosis of a mycotic aneurysm was made. A septic screen was performed and no source of infection was found. The patient underwent urgent abdominal aortic aneurysm repair on the next available list. At operation, an infrarenal aortic aneurysm was found. It was not of an inflammatory nature nor atherosclerotic. The rest of the laparotomy was normal. An 18 mm gelatin sealed, knitted Dacron polyester tube graft was used to replace the aneurysm. Postoperative recovery was unremarkable and the patient was discharged home eight days later.

Histological examination of the resected aneurysm specimen ruled out the presence of any connective tissue abnormality. A skin biopsy ruled out connective tissue disease.

\section{DISCUSSION}

This is a unique case of an abdominal aortic aneurysm in a young man of Caribbean descent. Abdominal aortic aneurysms are considered to be primarily a disease of the elderly and are uncommon before the sixth decade of life. ${ }^{1}$ They are rare in young patients
Summary points

- Abdominal aortic aneurysms are rare in young patients and less common in men of Afro-Caribbean descent.

- They can be associated with connective tissue diseases, especially in younger patients.

- There is a recent resurgence of mycotic aneurysms with intravenous drug abuse. A persistent fever without a known source in the presence of a newly discovered aneurysm should alert the physician to the possibility of a mycotic aneurysm.

- Patients can develop graft related complications including rupture of the graft as late as 19 years after surgery.

- There are claims of enhanced strength and stability of newer generation of grafts (like the one used in this patient). There has been no study to evaluate the long term durability of these newer grafts.

and less common in men of Afro-Caribbean descent. ${ }^{12}$ In this case there was a suspicion of connective tissue abnormality, connective tissue diseases being known to be associated with aortic aneurysms, especially in younger patients. ${ }^{3}$ Histological examination of the aneurysm, skin biopsy, and blood tests ruled out this possibility. However, the history of inguinal hernia repair and reputed Achilles tendonitis suggests that there may as yet be an undiagnosed connective tissue disorder.

A working diagnosis of a mycotic aneurysm was initially made. Osler in 1885 first coined the term mycotic aneurysm in his classic discussion of bacterial endocarditis. ${ }^{4}$ They occur when septic emboli lodge in either the lumen or the vasa vasorum of a blood vessel. This leads to degradation and thinning of the wall and subsequent dilatation.

Such aneurysms have become rare after the widespread use of aggressive antibiotic treatment for bacterial endocarditis. The diagnosis of an aortic mycotic aneurysm is often difficult. ${ }^{5} \mathrm{~A}$ persistent fever without a known source in the presence of a newly discovered aneurysm should alert the physician to the possibility of a mycotic aneurysm. Our patient remained apyrexial throughout. Treatment of mycotic aneurysms includes aggressive debridement of infected tissues, arterial bypass, and appropriate antibiotic therapy. Interestingly, there has been a recent resurgence of mycotic aneurysms with intravenous drug abuse becoming more prevalent. No such evidence existed in this case.

The question of long term durability of the Dacron graft remains. In this case a young man has had a Dacron graft replacement of his aorta at the age of 34 years. A long term follow up population based study in US that looked at 307 patients who underwent abdominal aortic aneurysm repair between 1957 and 1990 found that $9.4 \%$ of the patients developed a graft related complication over four decades. ${ }^{6}$ A review of the literature shows that there are 11 reported cases of aortic Dacron graft rupture between 1970 and 1996; this includes four involving knitted Dacron grafts. ${ }^{7}$ These ruptures were due to graft degeneration and occurred as late as 19 years after surgery. 
We used a "Gelsoft plus" type of Dacron graft made by Sulzer Vascutek Ltd, which is a new gelatin sealed koper knitted polyester graft. $^{8}$ The manufacturers claim that this new graft provides enhanced strength and radial stability. ${ }^{9}$ There are no independent studies to evaluate the long term durability of these newer grafts but a textile analysis performed on a similar 10 year old graft by the manufacturer showed that it had only dilated by $30 \%$ with no degenerative changes. ${ }^{10}$

In summary, the case highlights an unusual case of a young man with an abdominal aortic aneurysm of an unknown cause. The patient has a Dacron graft in place, the long term durability of which is not known. He will require long term follow up with the possibility of graft replacement in the future.

\section{Authors' affiliations}

B F Khan, Pennine Acute Hospitals NHS Trust, Royal Oldham Hospital Rochdale Road, Oldham

S Renton, S T Hussain, North West London Hospitals NHS Trust,

Northwick Park Hospital, Harrow, Middlesex

Correspondence to: Dr Bilal Firdaus Khan, Pennine Acute Hospitals NHS Trust, Royal Oldham Hospital, Rochdale Road, Oldham OL1 2JH, UK; bilalfirdauskhan@aol.com

Submitted 1 November 2002

Accepted 19 December 2002

\section{REFERENCES}

1 Muluk SC, Gertler JP, Brewster DC, et al. Presentation and patterns of aortic aneurysms in young patients. J Vasc Surg 1994;20:880-6, 887-8.

2 Costa M, Robbs JV. Abdominal aneurysms in a black population: clinicopathological study. Br J Surg 1986;73:554-8.

3 Wilmink AB, Quick CR, Hubbard CS, et al. The association between connective tissue laxity and the risk of an abdominal aortic aneurysm. Eur J Vasc Endovasc Surg 2000;20:290-5.

4 Akers DL Jr, Fowl RJ, Kempczinski RF. Mycotic aneurysm of the tibioperoneal trunk: case report and review of the literature. J Vasc Surg 1992:16:71-4.

5 Spencer B, Ronald B, Baker J, et al. Bacteriologic and surgical determinants of survival in patients with mycotic aneurysms. J Vasc Surg 1984;1:541-7.

6 Hallett JW Jr, Marshall DM, Petterson TM, et al. Graft-related complications after abdominal aortic aneurysm repair: reassurance from a 36-year population-based experience. J Vasc Surg 1997;25:277-84.

7 Wilson SE, Krug R, Mueller G, et al. Late disruption of Dacron aortic grafts. Ann Vasc Surg 1997;11:383-6.

8 Mary C, Marois Y, King MW, et al. In vitro and in vivo studies of a polyester arterial prosthesis with a warp-knitted sharkskin structure. $J$ Biomed Mater Res 1997;35:459-72.

9 Sulzer Vascutek Ltd. Available at: www.vascutek.com/index l.html.

10 McNeil G. Physical testing, microscopic examination and textile analysis report on a graft returned from the North Manchester General Hospital. Inchinnan, Renfrewshire, Scotland: Textile Department, Sulzer Vascutek, March 2000.

\section{Readers' favourite}

\section{Top 10}

Click on the "Top 10" button on the homepage

to see which are the best read articles each month

www.postgradmedj.com 